\section{Umfassendes Standardwerk der Schlafmedizin}

$E_{\text {n }}^{\text {in }}$ in kompetentes Autorenteam um ei- nen der bekanntesten Schlafmediziner aus dem HNO-Fach, Boris A. Stuck, Mannheim, hat ein schon lange fälliges Buch über die Praxis der Schlafmedizin verfasst. Denn 20\% der Bevölkerung leiden unter Schlafstörungen, über eine halbe Million Menschen in Deutschland nehmen regelmäßig Schlafmittel ein. Eine notwendige differenzierte schlafmedizinische Diagnostik als Voraussetzung für eine optimale langfristige Therapie erfolgt häufig nicht. Das vorgelegte moderne
Lehr- und Lernbuch hilft, diese Lücke zu schließen. Es dient zur Prüfungsvorbereitung zum Qualifikationsnachweis Somnologie der DGSM, aber auch als Ratgeber für die tägliche, praktische Tätigkeit in Klinik und Praxis. Grundlagen, Methoden, Diagnostik, Differenzialdiagnostik und Therapiemöglichkeiten werden umfassend erörtert. Alles, was klinisch relevant ist, wird mit Praxistipps, Fallbeispielen und Fragen zur Prüfungsvorbereitung dargestellt. Die inhaltliche und optische Gliederung ist klar und vorbildlich.

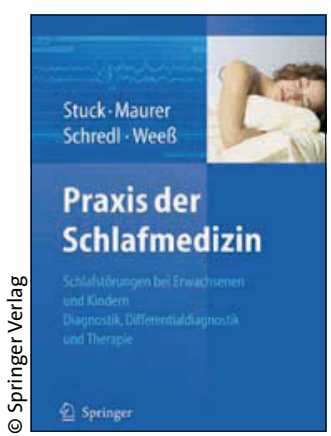

Stuck, B., Maurer, J.T., Schredl, M., Weeß, H.-G. Praxis der Schlafmedizin Springer Verlag 2010, 290 S, 65,95 Euro, ISBN: 978-3540-88699-0

Das Buch ist ein Muss für jeden schlafmedizinisch tätigen HNO-Arzt, ein Standardwerk für ein klinisch wichtiges Kapitel unseres Fachgebietes.

\title{
Psychosomatik-Wissen für den HNO-Arzt
}

Tinnitus und Schwindel nach Unfäl-

len werden immer häufiger als Symptome einer posttraumatischen Belastungsstörung (PTBS) eingeordnet, so dass auch der HNO-Arzt mit dieser Störung konfrontiert sein kann.

Unter PTBS werden verschiedene psychosomatische und psychische Störungen zusammengefasst, die als Langzeitfolgen nach dem belastenden Ereignis auftreten. Aber ist es immer angebracht, von einer PTBS zu reden? Nach WHO dürfen als PTBS nur psychische Störungen nach Katastrophenerlebnissen (z. B. Zugunglück bei Eschede, Tsu- nami im Urlaubsparadies) oder nach Folter, sexuellem Missbrauch, Vergewaltigungen oder Geiselhaft bezeichnet werden. Das Buch stellt gut dar, was PTBS alles umfasst, aber nicht so deutlich, was es eigentlich nicht ist. Der gutachterlich orientierte HNO-Arzt kommt dadurch etwas kurz, wenn er die Behauptung widerlegen möchte, dass ein Tinnitus nach Unfall als PTBS anzusehen ist. Das Buch bietet aber ansonsten - insbesondere dem psychosomatisch orientierten HNO-Arzt - fundierte und ausführliche Informationen zu allen Formen des Krankheitsbildes,

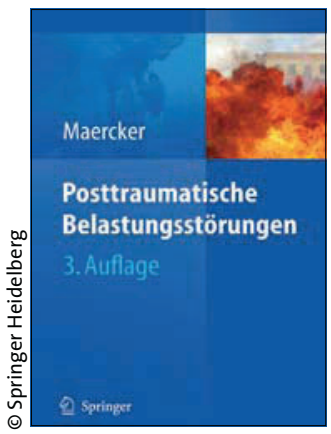

Maercker A. (Hrsg) Postraumatische Belastungsstörungen Springer Verlag, 2009, 522 S., 59,95 Euro, ISBN: 978-3-54088488-0

seiner Diagnosestellung und den Behandlungsmöglichkeiten, zu Akutinterventionen und dem Auftreten einer PTBS bei Kindern.

om

\section{Perspektiven der Psychoonkologie}

D ie Diagnose „Krebs“ trifft in Deutschland jährlich fast eine halbe Million Menschen. Über ein Drittel der Betroffenen hat daraufhin mit erheblichen psychischen Beeinträchtigungen zu kämpfen, die sich auch auf den Krankheitsverlauf und die Lebensqualität auswirken können.

Das Buch „Ressourcenorientierte Psychoonkologie" zeigt aktuelle Trends und Perspektiven der Psychoonkologie sowie der Onkologie, Palliativmedizin und
Neurobiologie. Es stellt psychotherapeutische und körperbezogene Interventionen unter einem explizit ressourcenorientierten Blickwinkel vor - mit Blick auf die inneren Potenziale der Betroffenen also.

Führende Experten der jeweiligen Fachgebiete geben Impulse für die Praxis und nicht zuletzt für die eigene Psychohygiene. Herausgegeben wird das Buch von Christa Diegelmann und Margarete Isermann, beide vom ID Ins-

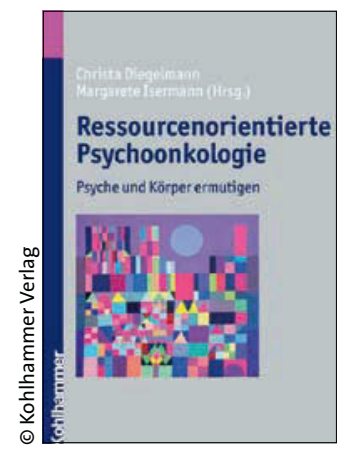

Diegelmann C. , Isermann $M$. (Hrsg.) Ressourcenorientierte Psychoonkologie Kohlhammer Verlag 2010, 29,80 Euro, 259 S., ISBN: 978-3-17020905-3

titut für Innovative Gesundheitskonzepte in Kassel. 